\title{
Elevated pre-treatment levels of plasma C-reactive protein are associated with poor prognosis after breast cancer: a cohort study
}

\author{
Kristine H Allin ${ }^{1,2}$, Børge G Nordestgaard ${ }^{1,2}$, Henrik Flyger ${ }^{3}$ and Stig E Bojesen ${ }^{1,2^{*}}$
}

\begin{abstract}
Introduction: We examined whether plasma C-reactive protein (CRP) levels at the time of diagnosis of breast cancer are associated with overall survival, disease-free survival, death from breast cancer, and recurrence of breast cancer.

Methods: We observed 2,910 women for up to seven years after they were diagnosed with invasive breast cancer (median follow-up time was three years). Plasma levels of high-sensitivity CRP were measured at the time of diagnosis and we assessed the association between CRP levels and risk of reduced overall and disease-free survival, death from breast cancer, and recurrence of breast cancer by using the Kaplan-Meier method and Cox proportional hazards regression. During follow-up, 383 women died (225 of whom died from breast cancer) and 118 women experienced recurrence of breast cancer.
\end{abstract}

Results: Elevated CRP levels across tertiles at the time of diagnosis were associated with reduced overall and disease-free survival and with increased risk of death from breast cancer (log-rank trend for all, $P<0.001$ ), but not with recurrence. The multifactor-adjusted hazard ratios (HR) of reduced overall survival among women in the middle and highest versus the lowest tertile of CRP were 1.30 ( $95 \% \mathrm{Cl}, 0.97$ to 1.73 ) and 1.94 (1.48 to 2.55), respectively. Corresponding HRs of reduced disease-free survival were 1.16 (0.89 to 1.50) and 1.76 (1.38 to 2.25) and of death from breast cancer 1.22 (0.84 to 1.78) and 1.66 (1.15 to 2.41). Dividing CRP levels into octiles resulted in a stepwise increased risk of reduced overall survival ( $P$ for trend $<0.001)$ and the multifactor-adjusted HR among women in the highest versus the lowest octile of CRP was 2.51 (1.53 to 4.12). Compared to women with CRP levels in the 0 to $25 \%$ percentile $(<0.78 \mathrm{mg} / \mathrm{L})$, the multifactor-adjusted HR of reduced overall survival among women with CRP levels $\geq 95 \%$ percentile ( $\geq 16.4 \mathrm{mg} / \mathrm{L}$ ) was 3.58 (2.36 to 5.42). Among women with HER2-positive tumours, the multifactor-adjusted HR of reduced overall survival for the highest versus the lowest tertile of CRP was 8.63 (2.04 to 36.4).

Conclusions: Elevated CRP levels at the time of diagnosis of breast cancer are associated with reduced overall and disease-free survival and with increased risk of death from breast cancer.

\section{Introduction}

Elevated plasma levels of C-reactive protein (CRP) may be associated with poor prognosis after breast cancer. CRP is a classical acute-phase protein displaying rapid and pronounced rise of its plasma concentration in response to acute inflammation, infection, and tissue damage [1,2]. Circulating levels of CRP are also

\footnotetext{
* Correspondence: stebo@heh.regionh.dk

'Department of Clinical Biochemistry Herlev Hospital, Copenhagen University

Hospital, Herlev Ringvej 75, Herlev, DK-2730, Denmark

Full list of author information is available at the end of the article
}

moderately elevated during chronic inflammatory diseases and cancer [3]. CRP is produced in the liver, predominantly under transcriptional control by the cytokine interleukin-6 originating from the site of pathology [4]. Many tumours arise at sites of chronic inflammation or they trigger inflammatory responses that result in the formation of an inflammatory microenvironment around the tumour [5-9]. In fact, it has recently been suggested that cancer-related inflammation may represent the seventh hallmark of cancer in addition to the six hallmarks identified by Hanahan and

\section{() Biomed Central}

(c) 2011 Allin et al.; licensee BioMed Central Ltd. This is an open access article distributed under the terms of the Creative Commons Attribution License (http://creativecommons.org/licenses/by/2.0), which permits unrestricted use, distribution, and reproduction in any medium, provided the original work is properly cited. 
Weinberg [6]. Since inflammation in the tumour microenvironment stimulates tumour growth, invasion, and metastasis, inflammation seems to favour invasion and metastasis more than to mount an effective host antitumour response [5-9].

Previous epidemiologic studies have reported that elevated CRP levels may be associated with poor prognosis of several types of solid cancers [10], including endometrial [11], cervical [12], colorectal, pancreatic, hepatocellular, esophageal, renal cell, bladder, prostate, ovarian, and non-small-cell lung cancer $[13,14]$. Although breast cancers rarely are characterized by significant histological inflammation, emerging evidence nevertheless suggests that inflammatory pathways also play an important role in breast cancer progression [15-20]. However, results from prospective epidemiologic studies are conflicting, with some studies showing an association between elevated CRP levels and poor prognosis [21-24] and others showing no association [25,26]. The largest study so far comprised 700 women treated successfully for early stage breast cancer and found that elevated levels of CRP measured two and a half years after the time of diagnosis were associated with reduced diseasefree and overall survival [23]. Thus, it is unclear whether CRP levels measured at the time of diagnosis are associated with breast cancer prognosis.

We used a prospective cohort study of 2,910 patients with invasive breast cancer to examine whether plasma CRP levels at the time of diagnosis of breast cancer are associated with overall survival, disease-free survival, death from breast cancer, and recurrence of breast cancer.

\section{Materials and methods Patients}

We studied patients with invasive breast cancer from the Copenhagen Breast Cancer Study, which is an ongoing cohort study of Danish breast cancer patients. Since January 2002, all patients that are referred to the Department of Breast Surgery, Herlev Hospital, Copenhagen University Hospital with suspected breast cancer are asked to participate in the study (participation rate 93\%). At study enrolment the patients answer a questionnaire regarding sociodemographic factors, lifestyle factors, anthropometric characteristics, medical history, and family history of cancer. Furthermore, they give blood for measurements of biochemical parameters.

In the present study, we included patients who were diagnosed with breast cancer in the period 1 January 2002 until 31 January 2009 ( $n=3,634$ ) (Figure 1). Since our intention was to study the association between CRP levels and prognosis among women with invasive breast cancer, we excluded patients without a measurement of CRP ( $\mathrm{n}=131)$, men $(\mathrm{n}=23)$, and women with in situ breast cancer $(\mathrm{n}=135)$. To avoid potential bias by the influence of surgery on CRP levels, we also excluded women who underwent breast cancer surgery before blood sampling $(n=137)$. Finally, to ensure that plasma CRP was measured at the time of diagnosis, we excluded women who had plasma CRP measured more than 30 days before or after the date of diagnosis of breast cancer $(\mathrm{n}=298)$. Thus, we included 2,910 women aged 26 to 99 years in the analysis of overall survival. For the analysis of disease-free survival and recurrence, we additionally excluded 72 women for whom information about recurrence was missing, 15 individuals who, according to the register, had recurrence less than 90 days after their date of diagnosis, and 40 women who had distant metastasis at diagnosis. The latter two groups were excluded to ensure that the registered recurrences were indeed true recurrences. Thus, 2,783 women aged 26 to 99 years were included in the analysis of disease-free survival and recurrence. For the analysis of death from breast cancer, we excluded 38 women who were enrolled after 31 December 2008, since we did not have information about cause-specific death after this date. Thus, we included 2,872 women aged 26 to 99 years in the analysis of death from breast cancer (Figure 1). Follow-up time for each patient began at blood sampling and ended for overall survival at occurrence of death, emigration, or May 2009, whichever came first. For disease-free survival and recurrence, follow-up time ended at occurrence of recurrence, death, emigration, or May 2009, whichever came first. For death from breast cancer, follow-up time ended at death from breast cancer, death from other causes, emigration, or December 2008, whichever came first. The median follow-up period was three years (range $=0$ to 7 years). During follow-up, 383 women died, 225 women died from breast cancer, 118 women experienced recurrence of breast cancer, and 4 women emigrated.

\section{Register information}

Information about diagnoses and prognostic tumour characteristics (tumour size, lymph node status, presence of distant metastases, tumour grade, and estrogen receptor, progesterone receptor, and HER2 status) of index breast cancers was obtained from the national Danish Breast Cancer Cooperative Group (DBCG) [27]. Diagnoses and prognostic tumour characteristics were pathologically verified by clinical pathologists. Date of recurrence was obtained from DBCG and date of death and emigration was obtained from the national Danish Civil Registration System, which is $100 \%$ complete. Information about death from breast cancer was obtained from the National Danish Causes of Death Registry in which all death certificates in Denmark are registered. Danish death certificates register courses of 


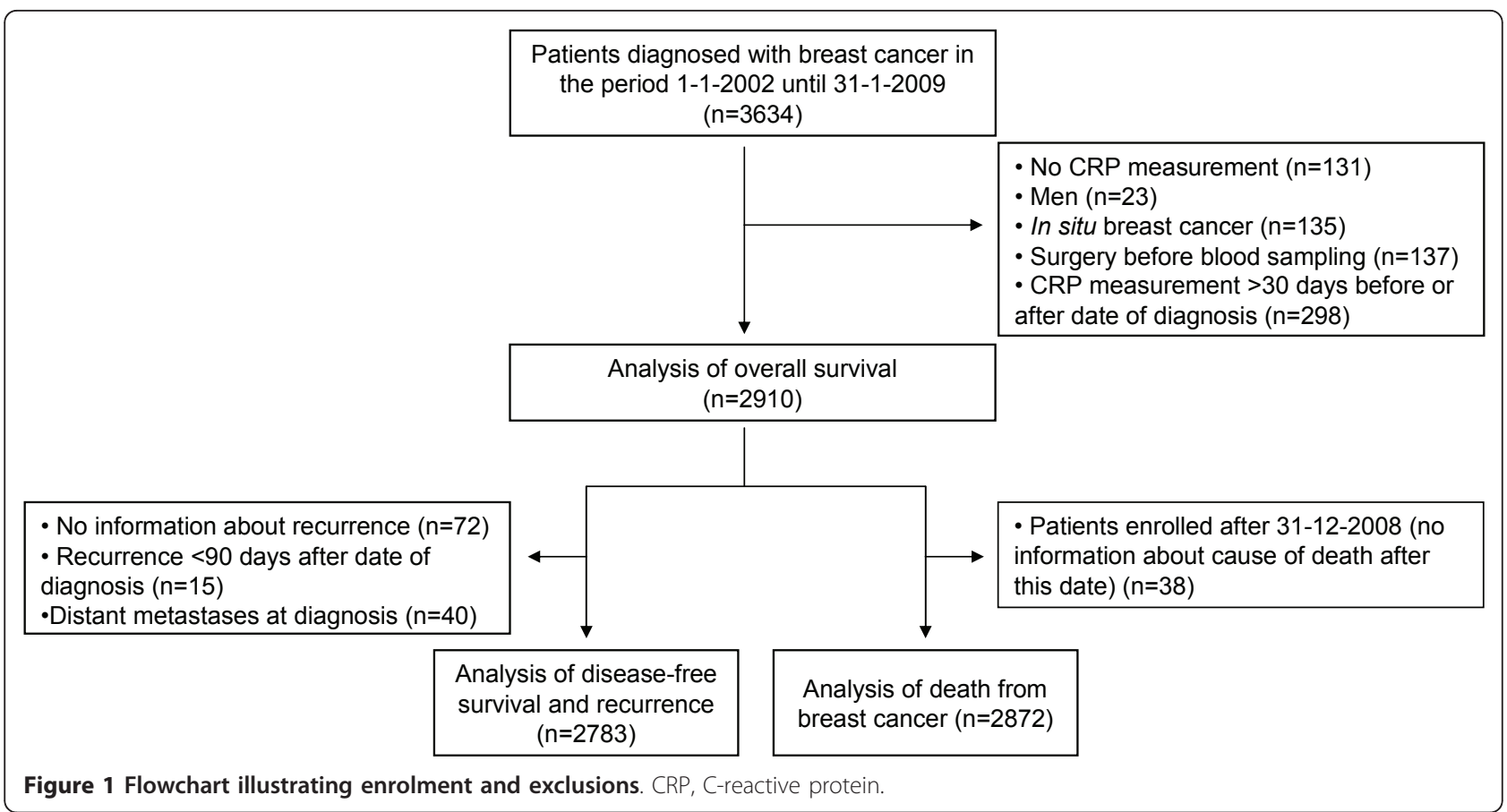

diseases which lead to the death, with the underlying cause of death being the disease which started this course. In the present study, patients who were registered with the World Health Organization (WHO) International Classification of Diseases (ICD), Tenth Revision (ICD-10) codes C50.0 to C50.9 as the underlying cause of death were recorded as having died from breast cancer.

Information about diagnosis of cardiovascular diseases was obtained from the national Danish Patient Registry which all public and private hospitals in Denmark report to. Diagnoses were classified according to the WHO ICD, Eighth Revision (ICD-8 codes 410 to 414 and 432 to 435) or Tenth Revision (ICD-10 codes I20 to I25 and I63 to I64 and G45).

\section{Ethics}

The ethical committee of Copenhagen and Frederiksberg, Denmark approved the study (KA-02152). All patients gave written informed consent.

\section{CRP analysis}

We determined the plasma CRP levels in fresh plasma samples by using a high-sensitivity nephelometry assay (CardioPhase hsCRP, code OQIY21, Dade Behring, Deerfield, IL, USA) performed on a Behring Nephelometer or a high-sensitivity turbidimetry assay (Code LX001, Dako, Glostrup, Denmark) performed on a Konelab 60 analyzer. All analyses were performed in the same laboratory at the Department of Clinical
Biochemistry, Herlev Hospital, Copenhagen University Hospital. The detection limit was $0.17 \mathrm{mg} / \mathrm{L}$ for the nephelometry assay and $0.20 \mathrm{mg} / \mathrm{L}$ for the turbidimetry assay. Prior to the switch from the nephelometry to the turbidimetry assay, the two assays were compared by measuring plasma CRP with each method in 29 individuals. Spearman rank correlation coefficient between the two CRP assays was 0.96 , and a Wilcoxon signedrank test for difference between the two assays yielded a $P$-value of 0.87. If measured CRP changed over calendar time, due to analytical fluctuations, this could bias our risk estimates, but we found no evidence for an interaction between CRP level and date of measurement (= diagnosis). When statistical analyses were run separately by assay, the results were similar; we, therefore, only present combined results in the paper. The assays were assessed daily for precision by using internal controls for each assay (coefficient of variation was $3 \%$ at a level of $1.7 \mathrm{mg} / \mathrm{L}$ for the nephelometry assay and $6 \%$ at a level of $2.1 \mathrm{mg} / \mathrm{L}$ for the turbidimetry assay) and monthly for accuracy through a Scandinavian quality control program.

\section{Statistical analysis}

The data were analyzed using STATA statistical software (version 10.1; StataCorp, College Station, TX, USA), and a two-sided $P$-value less than 0.05 was considered statistically significant. On the basis of the measurements of plasma CRP with the nephelometry and turbidemetry assays, we divided patients into assay- 
specific tertiles of CRP and thereupon combined these assay-specific tertiles into common tertiles of CRP. To test the robustness of our findings, we used the same approach to divide patients into octiles of CRP, and to examine extremely high CRP levels, we divided patients into five groups of CRP levels ( 0 to $25 \%$ percentile, 25 to $50 \%$ percentile, 50 to $75 \%$ percentile, 75 to $95 \%$ percentile, and $\geq 95 \%$ percentile). The decision to examine tertiles as well as robustness using octiles, and extreme phenotypes was decided a priori, as we have done previously in similar studies, but for other questions [28,29].

To test whether covariates differed across tertiles of plasma CRP, Kruskal-Wallis one-way analysis of variance was used for continuous covariates and the chi square test was used for categorical covariates. We used the Kaplan-Meier method to plot overall survival, disease-free survival, or cumulative incidence of death from breast cancer and recurrence against follow-up time and used the log-rank test to test for differences across tertiles of CRP. Cox proportional hazards regression models were used to estimate hazard ratios (HRs) and 95\% confidence intervals (CIs) of reduced overall and disease-free survival, death from breast cancer, and recurrence as a function of elevated plasma CRP levels. Patients were censored at their date of death (or emigration), dates that are $100 \%$ complete in the Danish registries. We used a model that included age at diagnosis only (Model 1), a multifactor-adjusted model that included age at diagnosis, tumour size $(\leq 20 \mathrm{~mm}, 20$ to $50 \mathrm{~mm},>50 \mathrm{~mm}$, or unknown), lymph node status (negative, positive, or unknown), presence of distant metastases (yes, no, or unknown), tumour grade (well differentiated, moderately differentiated, poorly/un-differentiated, or unknown), estrogen receptor status (positive, negative, or unknown), progesterone receptor status (positive, negative, or unknown), and HER2 status (negative, positive, or unknown) (Model 2), and a multifactor-adjusted model that included the above-mentioned covariates and the lifestyle factors smoking $(<10$, 10 to $20, \geq 20$ cigarettes per day, or unknown), alcohol consumption ( $\leq 168$ or $>168$ grams per week, or unknown), and body mass index $(<18.5,18.5$ to $24.9,25$ to 29.9 , or $\geq 30.0 \mathrm{~kg} / \mathrm{m}^{2}$, or unknown) as well as menopause status (pre-menopause, post-menopause, or unknown) and cardiovascular disease (yes, no, or unknown) (Model 3). For trend tests in log-rank and Cox statistics, the CRP groups were coded 1, 2, 3, and so on, corresponding to CRP tertiles and octiles.

For Cox proportional hazards regression analyses, we assessed the assumption of proportional hazards graphically by plotting $\log$ (cumulative hazard) as a function of follow-up time. We detected no major violations of the proportional hazards assumption. Interactions between tertiles of plasma CRP and covariates in the regression models was tested for by computing a likelihood ratio test comparing the statistical fit of models with and without a two-factor interaction term.

\section{Results}

Baseline characteristics of the patients by CRP tertiles at the time of diagnosis of breast cancer are shown in Table 1. Levels of CRP were associated with several of these characteristics. The median duration from date of diagnosis to date of blood sampling was seven days (IQR, two to nine days). Table 2 shows the risk of reduced overall and disease-free survival, death from breast cancer, and recurrence by age at diagnosis, prognostic tumour characteristics, lifestyle factors, menopause status, and presence of cardiovascular disease. Causes of deaths in the present cohort were breast cancer (64\%), other cancer (11\%), cardiovascular disease (11\%), respiratory disease (4\%), other disease (11\%), and unknown cause (1\%).

\section{Overall survival}

The overall survival among breast cancer patients decreased with increasing levels of CRP (Figure 2A; logrank trend, $P<0.001)$. The five-year survival rates were $90 \%, 81 \%$, and $74 \%$ among women in the lowest, middle, and highest tertile of CRP. Corresponding age-adjusted HRs were $1.00,1.39$ (95\% CI, 1.04 to 1.85 ), and 2.13 (1.63 to 2.79 ); $P$ for trend $<0.001$ (Figure 3 ). Multifactorial adjustment for prognostic tumour characteristics (Model 2) slightly reduced the HRs to $1.00,1.28$ (0.96 to 1.70$)$, and 1.91 (1.46 to 2.50 ); $P$ for trend $<0.001$. Additional adjustment for lifestyle factors, menopause status, and cardiovascular disease (Model 3) resulted in similar HRs of 1.00, 1.29 (0.96 to 1.72 ), and 1.84 (1.39 to 2.45 ); $P$ for trend $<0.001$.

\section{Disease-free survival}

The disease-free survival among breast cancer patients decreased with increasing levels of CRP (Figure 2B; logrank trend, $P<0.001)$. The five-year disease-free survival rates were $87 \%, 80 \%$, and $74 \%$ among women in the lowest, middle, and highest tertile of CRP. Corresponding age-adjusted HRs were 1.00, 1.25 (95\% CI, 0.96 to 1.63), and 1.82 (1.42 to 2.34); $P$ for trend $<0.001$ (Figure 3). Multifactorial adjustment for prognostic tumour characteristics (Model 2) slightly reduced the HRs to $1.00,1.16$ (0.89 to 1.51 ), and 1.70 (1.32 to 2.18 ); $P$ for trend $<0.001$. Additional adjustment for lifestyle factors, menopause status, and cardiovascular disease (Model 3) resulted in similar HRs of $1.00,1.16$ (0.88 to 1.52$)$, and 1.65 (1.26 to 2.15 ); $P$ for trend $<0.001$.

\section{Death from breast cancer}

The cumulative incidence of death from breast cancer among breast cancer patients increased with increasing 
Table 1 Baseline characteristics of breast cancer patients by plasma levels of C-reactive protein

\begin{tabular}{|c|c|c|c|c|}
\hline & \multicolumn{3}{|c|}{ Tertiles of C-reactive protein (mg/L) } & \multirow[b]{2}{*}{$P$} \\
\hline & $\begin{array}{c}1 \text { st } \\
(<1.04)\end{array}$ & $\begin{array}{c}2 \mathrm{nd} \\
(1.04 \text { to } 3.24)\end{array}$ & $\begin{array}{c}3 \mathrm{rd} \\
(>3.24)\end{array}$ & \\
\hline No. of patients & 975 & 966 & 969 & \\
\hline Median age, years (IQR) & 59 (48 to 68 ) & 64 (55 to 73 ) & 65 (56 to 74$)$ & $<0.001$ \\
\hline \multicolumn{5}{|l|}{ Tumour size, No. (\%) } \\
\hline$\leq 20 \mathrm{~mm}$ & $587(60)$ & $487(50)$ & $427(44)$ & \\
\hline 20 to $50 \mathrm{~mm}$ & $297(30)$ & $377(39)$ & $384(40)$ & \\
\hline$>50 \mathrm{~mm}$ & $25(3)$ & $31(3)$ & $47(5)$ & $<0.001$ \\
\hline Unknown & $66(7)$ & $71(7)$ & $111(11)$ & \\
\hline \multicolumn{5}{|l|}{ Lymph node status, No. (\%) } \\
\hline Lymph node negative & $459(47)$ & $453(47)$ & $442(44)$ & \\
\hline Lymph node positive & $451(46)$ & $443(46)$ & $441(46)$ & 0.74 \\
\hline Unknown & $65(7)$ & $70(7)$ & $106(11)$ & \\
\hline \multicolumn{5}{|l|}{ Distant metastases, No. (\%) } \\
\hline No & $942(97)$ & $929(96)$ & $909(94)$ & \\
\hline Yes & $6(1)$ & $7(1)$ & $27(3)$ & $<0.001$ \\
\hline Unknown & $27(3)$ & $30(3)$ & $33(3)$ & \\
\hline \multicolumn{5}{|l|}{ Tumour grade, No. (\%) } \\
\hline Well differentiated & $248(25)$ & $243(25)$ & $194(20)$ & \\
\hline Moderately differentiated & $431(44)$ & $392(41)$ & $439(45)$ & \\
\hline Poorly/un-differentiated & $158(16)$ & $193(20)$ & $154(16)$ & 0.006 \\
\hline Unknown & $138(14)$ & $138(14)$ & $182(19)$ & \\
\hline \multicolumn{5}{|l|}{ Estrogen receptor status, No. (\%) } \\
\hline Positive & $766(79)$ & $744(77)$ & $723(75)$ & \\
\hline Negative & $146(15)$ & $150(16)$ & $140(14)$ & 0.90 \\
\hline Unknown & $63(6)$ & $72(7)$ & $106(11)$ & \\
\hline \multicolumn{5}{|c|}{ Progesterone receptor status, No. (\%) } \\
\hline Positive & $492(50)$ & $451(47)$ & $440(45)$ & \\
\hline Negative & $243(25)$ & $286(30)$ & $258(27)$ & 0.07 \\
\hline Unknown & $240(25)$ & $229(24)$ & $271(28)$ & \\
\hline \multicolumn{5}{|l|}{ HER2 status, No. (\%) } \\
\hline Positive & $279(29)$ & $260(27)$ & $268(28)$ & \\
\hline Negative & $82(8)$ & $68(7)$ & $70(7)$ & 0.76 \\
\hline Unknown & $614(63)$ & $638(66)$ & $631(65)$ & \\
\hline \multicolumn{5}{|c|}{ Cigarettes smoked per day, No. (\%) } \\
\hline$<10$ & $689(71)$ & $624(65)$ & $531(55)$ & \\
\hline 10 to 20 & $91(9)$ & $110(11)$ & $141(15)$ & \\
\hline$\geq 20$ & $61(6)$ & $80(8)$ & $110(11)$ & $<0.001$ \\
\hline Unknown & $134(14)$ & $152(16)$ & $187(19)$ & \\
\hline \multicolumn{5}{|c|}{ Alcohol consumption in grams per week*, No. (\%) } \\
\hline$\leq 168$ & $786(81)$ & $743(77)$ & $702(72)$ & \\
\hline$>168$ & $66(7)$ & $82(8)$ & $86(9)$ & 0.08 \\
\hline Unknown & $123(13)$ & $141(15)$ & $181(19)$ & \\
\hline \multicolumn{5}{|c|}{ Body mass index in kg/m ${ }^{2 \dagger}$, No. (\%) } \\
\hline$<18.5$ & $46(5)$ & $16(2)$ & $15(2)$ & \\
\hline 18.5 to 24.9 & $554(57)$ & $367(38)$ & $255(26)$ & \\
\hline 25 to 29.9 & $133(14)$ & $241(25)$ & $242(25)$ & \\
\hline$\geq 30$ & $18(2)$ & $75(8)$ & $138(14)$ & $<0.001$ \\
\hline Unknown & $224(23)$ & $267(28)$ & $319(33)$ & \\
\hline \multicolumn{5}{|l|}{ Menopause status, No (\%) } \\
\hline Post menopause & $640(66)$ & $795(82)$ & $825(85)$ & \\
\hline Pre menopause & 325 (33) & $159(16)$ & $130(13)$ & $<0.001$ \\
\hline
\end{tabular}


Table 1 Baseline characteristics of breast cancer patients by plasma levels of C-reactive protein (Continued)

\begin{tabular}{|c|c|c|c|c|}
\hline Unknown & $10(1)$ & $12(1)$ & $14(1)$ & \\
\hline \multicolumn{5}{|c|}{ Cardiovascular disease, No (\%) } \\
\hline No & $910(93)$ & $869(90)$ & $847(87)$ & \\
\hline Yes & $38(4)$ & $60(6)$ & $78(8)$ & $<0.001$ \\
\hline Unknown & $27(3)$ & $37(4)$ & $44(5)$ & \\
\hline
\end{tabular}

Blood samples were drawn and CRP measured at time of diagnosis of breast cancer.

$P$-values were calculated using $\chi^{2}$ tests for categorical variables and Kruskal-Wallis one-way analysis of variance tests for continuous variables. For the evaluation of $P$-values, patients with unknown values were excluded.

$\mathrm{IQR}=$ interquartile range.

*Cut-off values are based on the recommendation from of the Danish National Board of Health

${ }^{+}$Body mass index was calculated as weight in kilograms divided by height in meters squared.

levels of CRP (Figure 2C; log-rank trend, $P<0.001$ ) reaching $11 \%, 19 \%$, and $20 \%$ among women in the lowest, middle, and highest tertile of CRP at the end of follow-up. Corresponding age adjusted HRs were 1.00, 1.41 (95\% CI, 0.98 to 2.04 ), and 2.12 (1.50 to 2.99); $P$ for trend $<0.001$ (Figure 3). Multifactorial adjustment for prognostic tumour characteristics (Model 2) slightly reduced the HRs to $1.00,1.22$ (0.84 to 1.76 ), and 1.78 (1.26 to 2.52$) ; P$ for trend $=0.001$. Additional adjustment for lifestyle factors, menopause status, and cardiovascular disease (Model 3) resulted in similar HRs of $1.00,1.22$ (0.84 to 1.78 ), and 1.66 (1.15 to 2.41$)$; $P$ for trend $=0.005$.

\section{Recurrence}

The cumulative incidence of recurrence among breast cancer patients was highest among women in the highest tertile of CRP, but the incidence of recurrence did not increase stepwise with increasing levels of CRP (Figure 2D; log-rank trend, $P=0.24$ ). The cumulative incidence of recurrence at the end of follow-up was $7 \%, 6 \%$, and $10 \%$ among women in the lowest, middle, and highest tertile of CRP. Corresponding age adjusted HRs were $1.00,1.11$ (95\% CI, 0.70 to 1.76 ), and 1.53 (0.98 to 2.37); $P$ for trend $=0.06$ (Figure 3 ). The HRs remained similar after multifactor-adjustment for prognostic tumour characteristics and additional adjustment for lifestyle factors, menopause status, and cardiovascular disease.

\section{Sensitivity analyses}

Dividing CRP levels into octiles also resulted in a stepwise increased risk of reduced overall survival (Figure 4; $P$ for trend $<0.001$ ). The multifactor-adjusted HR of reduced overall survival among women in the highest versus the lowest octile of CRP was 2.50 (95\% CI, 1.52 to 4.10 ) (Figure 4). Compared to women with CRP levels in the 0 to $25 \%$ percentile $(<0.78 \mathrm{mg} / \mathrm{L})$, the multifactor-adjusted HR of reduced overall survival was 3.59 (2.37 to 5.44) among women with CRP levels $\geq 95 \%$ percentile ( $\geq 16.4 \mathrm{mg} / \mathrm{L})$ (Figure 4$)$.
In sub-analyses stratified for age at diagnosis and prognostic tumour characteristics, elevated CRP levels across tertiles were associated with reduced overall survival irrespective of presence of distant metastases, and estrogen and progesterone receptor status (Table 3). Furthermore, elevated CRP levels were associated with reduced overall survival separately in women above 57 years, with small and middle-sized tumours, positive lymph node status, moderately and poorly/undifferentiated tumours, and HER2 positive tumours. The multifactor-adjusted HR of reduced overall survival for the highest versus the lowest tertile of CRP was 8.63 (2.04 to 36.4) among women with HER2 positive tumours. However, tests of interaction between the CRP tertiles and the prognostic factors were all non-significant, suggesting that elevated CRP levels are associated with similarly reduced overall survival irrespective of age at diagnosis, tumour size, lymph node status, presence of distant metastases, tumour grade, and estrogen receptor, progesterone receptor, and HER2 status (Table 3). Exclusion of patients with CRP values above $40 \mathrm{mg} / \mathrm{L}$, which likely reflect bacterial infection, resulted in similar hazard ratios for all endpoints (data not shown).

\section{Discussion}

Using a prospective cohort study of 2,910 Danish women with invasive breast cancer, we have demonstrated that elevated CRP levels at the time of diagnosis of breast cancer were associated with reduced overall and disease-free survival and with increased risk of death from breast cancer. These are novel observations.

Mechanistically, three components might explain the observed association between elevated CRP levels and poor breast cancer prognosis. First, tumour cell behavior: plasma CRP levels may reflect the aggressiveness of the tumour, that is, plasma CRP levels might sum up some prognostic information of well-known tumour characteristics, such as tumour stage and grade. In the present study, elevated CRP levels were indeed associated with larger tumour size, presence of distant metastases, and lower tumour grade (although CRP was 
Table 2 Prognoses after diagnosis of breast cancer by tumour characteristics and lifestyle factors

\begin{tabular}{|c|c|c|c|c|}
\hline \multirow{2}{*}{ Prognostic and lifestyle factors } & Overall survival & Disease-free survival & Death from breast cancer & Recurrence \\
\hline & \multicolumn{4}{|c|}{ Hazard ratio $(95 \% \mathrm{Cl})$} \\
\hline \multicolumn{5}{|l|}{ Age, years } \\
\hline$<57$ & 1.00 & 1.00 & 1.00 & 1.00 \\
\hline 57 to 69 & 1.51 (1.12 to 2.03$)$ & $1.19(0.90$ to 1.56$)$ & 1.13 (0.79 to 1.62$)$ & 0.61 (0.40 to 0.93$)$ \\
\hline$\geq 69$ & $3.20(2.45$ to 4.18$)$ & 2.25 (1.76 to 2.87$)$ & 2.05 (1.49 to 2.82$)$ & 0.56 (0.36 to 0.88$)$ \\
\hline \multicolumn{5}{|l|}{ Tumour size, mm } \\
\hline$\leq 20$ & 1.00 & 1.00 & 1.00 & 1.00 \\
\hline 20 to 50 & 2.39 (1.89 to 3.01$)$ & 2.12 (1.72 to 2.62$)$ & $3.27(2.37$ to 4.51$)$ & 1.49 (1.03 to 2.17$)$ \\
\hline$>50$ & 3.48 (2.22 to 5.44$)$ & 2.44 (1.53 to 3.90$)$ & 4.27 (2.33 to 7.82$)$ & 0.95 (0.30 to 3.03 ) \\
\hline \multicolumn{5}{|l|}{ Lymph node status } \\
\hline Lymph node negative & 1.00 & 1.00 & 1.00 & 1.00 \\
\hline Lymph node positive & 1.47 (1.18 to 1.84$)$ & $1.48(1.21$ to 1.82$)$ & 2.49 (1.81 to 3.43$)$ & 2.08 (1.41 to 3.07 ) \\
\hline \multicolumn{5}{|l|}{ Distant metastases } \\
\hline No & 1.00 & NA & 1.00 & NA \\
\hline Yes & 8.57 (5.61 to 13.1$)$ & NA & 12.32 (7.66 to 19.80$)$ & NA \\
\hline \multicolumn{5}{|l|}{ Histopathological grade } \\
\hline Well differentiated & 1.00 & 1.00 & 1.00 & 1.00 \\
\hline Moderately differentiated & 1.64 (1.19 to 2.26$)$ & 1.57 (1.16 to 2.11$)$ & $2.52(1.53$ to 4.13$)$ & 1.94 (1.05 to 3.59$)$ \\
\hline Poorly/un-differentiated & 2.76 (1.96 to 3.90$)$ & 3.00 (2.18 to 4.12$)$ & 5.28 (3.17 to 8.79$)$ & 5.32 (2.87 to 9.87$)$ \\
\hline \multicolumn{5}{|l|}{ Estrogen receptor status } \\
\hline Positive & 1.00 & 1.00 & 1.00 & 1.00 \\
\hline Negative & 2.09 (1.63 to 2.66$)$ & 1.98 (1.57 to 2.49$)$ & 3.03 (2.24 to 4.10$)$ & 2.31 (1.54 to 3.47$)$ \\
\hline \multicolumn{5}{|l|}{ Progesterone receptor status } \\
\hline Positive & 1.00 & 1.00 & 1.00 & 1.00 \\
\hline Negative & $2.32(1.77$ to 3.05$)$ & $2.02(1.58$ to 2.60$)$ & $3.28(2.25$ to 4.77$)$ & 1.91 (1.23 to 2.95$)$ \\
\hline \multicolumn{5}{|l|}{ HER2 receptor status } \\
\hline Positive & 1.00 & 1.00 & 1.00 & 1.00 \\
\hline Negative & 2.46 (1.38 to 4.39$)$ & 2.06 (1.21 to 3.52$)$ & $2.72(1.30$ to 5.69$)$ & 1.49 (0.58 to 3.85$)$ \\
\hline \multicolumn{5}{|l|}{ No. of cigarettes smoked per day } \\
\hline$<10$ & 1.00 & 1.00 & 1.00 & 1.00 \\
\hline 10 to 20 & 1.30 (0.97 to 1.75$)$ & 1.15 (0.86 to 1.54$)$ & 1.55 (1.09 to 2.21$)$ & 0.96 (0.55 to 1.66$)$ \\
\hline$\geq 20$ & 1.06 (0.74 to 1.51$)$ & 0.96 (0.68 to 1.36$)$ & 0.81 (0.49 to 1.37$)$ & $0.73(0.37$ to 1.45$)$ \\
\hline \multicolumn{5}{|c|}{ Alcohol consumption in grams per week* } \\
\hline$\leq 168$ & 1.00 & 1.00 & 1.00 & 1.00 \\
\hline$>168$ & 0.79 (0.53 to 1.19$)$ & $0.72(0.48$ to 1.08$)$ & 0.96 (0.59 to 1.55$)$ & $0.83(0.42$ to 1.65$)$ \\
\hline \multicolumn{5}{|l|}{ Body mass index in $\mathrm{kg} / \mathrm{m}^{2+}$} \\
\hline$<18.5$ & 2.21 (1.37 to 3.57$)$ & 2.09 (1.31 to 3.32$)$ & 1.10 (0.48 to 2.52$)$ & 0.32 (0.04 to 2.29$)$ \\
\hline 18.5 to 24.9 & 1.00 & 1.00 & 1.00 & 1.00 \\
\hline 25 to 29.9 & $1.06(0.80$ to 1.40$)$ & 1.09 (0.84 to 1.42$)$ & $1.05(0.74$ to 1.49$)$ & 1.16 (0.74 to 1.82$)$ \\
\hline$\geq 30$ & 1.45 (1.01 to 2.09$)$ & 1.58 (1.13 to 2.21$)$ & $1.62(1.05$ to 2.52$)$ & 1.88 (1.08 to 3.25$)$ \\
\hline \multicolumn{5}{|l|}{ Menopause status, No (\%) } \\
\hline Post menopause & 1.00 & 1.00 & 1.00 & 1.00 \\
\hline Pre menopause & $0.36(0.26$ to 0.51$)$ & 0.56 (0.42 to 0.74$)$ & 0.52 (0.36 to 0.77$)$ & 1.64 (1.12 to 2.42$)$ \\
\hline \multicolumn{5}{|l|}{ Cardiovascular disease, No (\%) } \\
\hline No & 1.00 & 1.00 & 1.00 & 1.00 \\
\hline Yes & 2.46 (1.77 to 3.42$)$ & 2.32 (1.67 to 3.22$)$ & $1.70(1.03$ to 2.79$)$ & 1.45 (0.71 to 2.98$)$ \\
\hline
\end{tabular}

*Cut-off values are based on the recommendation from of the Danish National Board of Health.

${ }^{+}$Body mass index was calculated as weight in kilograms divided by height in meters squared. Hazard ratios are unadjusted.

NA: not applicable. 


\section{A. Overall survival after a breast cancer diagnosis}

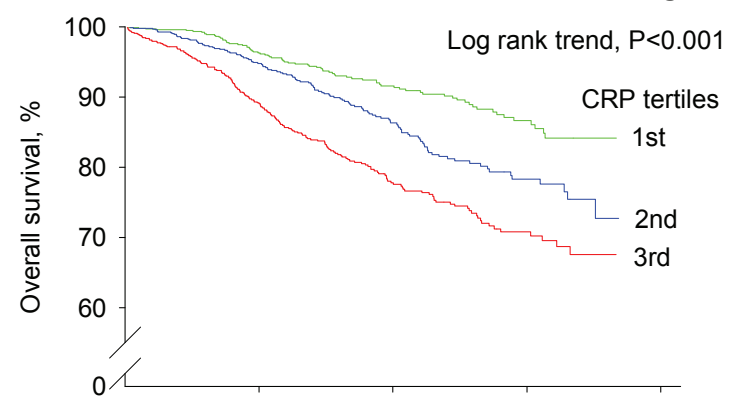

\section{B. Disease-free survival after a breast cancer diagnosis}

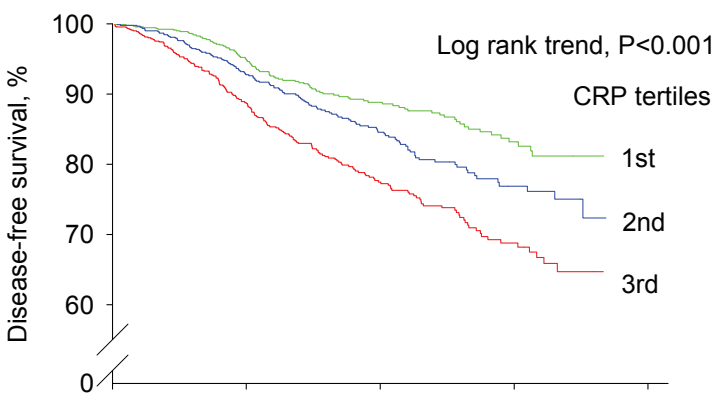

C. Death from breast cancer after a breast cancer diagnosis

\section{Recurrence after a breast cancer diagnosis}

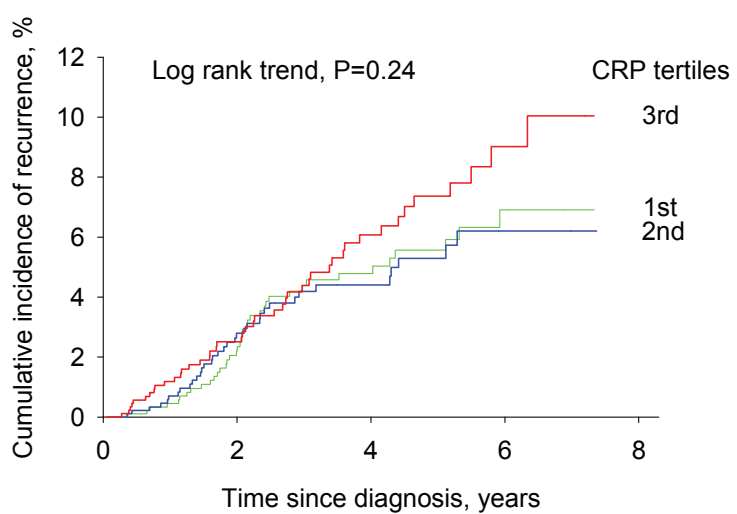

Figure 2 Overall survival (A), disease-free survival (B), death from breast cancer (C), and cumulative incidence of recurrence after a breast cancer diagnosis (D). Results are shown by tertiles of plasma C-reactive protein (CRP) and are based on 2,910 women from the Copenhagen Breast Cancer Study who were observed for up to seven years.

not linearly associated with tumour grade), and these prognostic factors were associated with poor prognosis. Second, adjacent inflammation: plasma CRP levels might express the magnitude and the nature of any inflammation in the breast tumour microenvironment. Inflammatory pathways play important roles in all stages of tumourigenesis, including tumour initiation and promotion, malignant transformation, tumour invasion, and metastasis [3-6]. Thus, solid tumours typically trigger inflammatory responses that result in the formation of a pro-tumourigenic and pro-angiogenic microenvironment around the tumour. Immune and inflammatory cells in the tumour microenvironment interact with malignant cells in a complicated fashion, the net result of which is stimulation of tumour growth, invasion, and metastasis [5-9]. Despite the fact that breast cancers rarely are characterized by significant histological inflammation, inflammation might also play a role in breast cancer prognosis [15-20]. Thus, macrophage infiltration into invasive breast carcinomas was associated with high vascularity of the breast tumour as well as with reduced recurrence-free and overall survival [18], and targeting of cancer associated fibroblasts resulted in favourable changes of the immune tumour microenvironment and improved anti-metastatic effects of doxorubicin chemotherapy in a murine model of metastatic breast cancer [19]. Furthermore, a recently published study showed that blockade of the IL-8 receptor selectively targets breast cancer stem cells and retards tumour growth and reduces metastasis [17]. Third, host behaviour: plasma CRP levels may outline the general health of the woman at the time of diagnosis of breast cancer. In the present study, elevated CRP levels were indeed associated with smoking and elevated body mass index at the time of diagnosis. However, whereas smoking was not associated with poor breast cancer prognosis, body mass index $<18.5$ or $\geq 30$ at the time of diagnosis was associated with poor prognosis. Previous studies have shown that elevated CRP levels are associated with allcause mortality in the general population $[29,30]$. Thus, it is possible that the present finding of an association between elevated CRP levels at the time of diagnosis of 


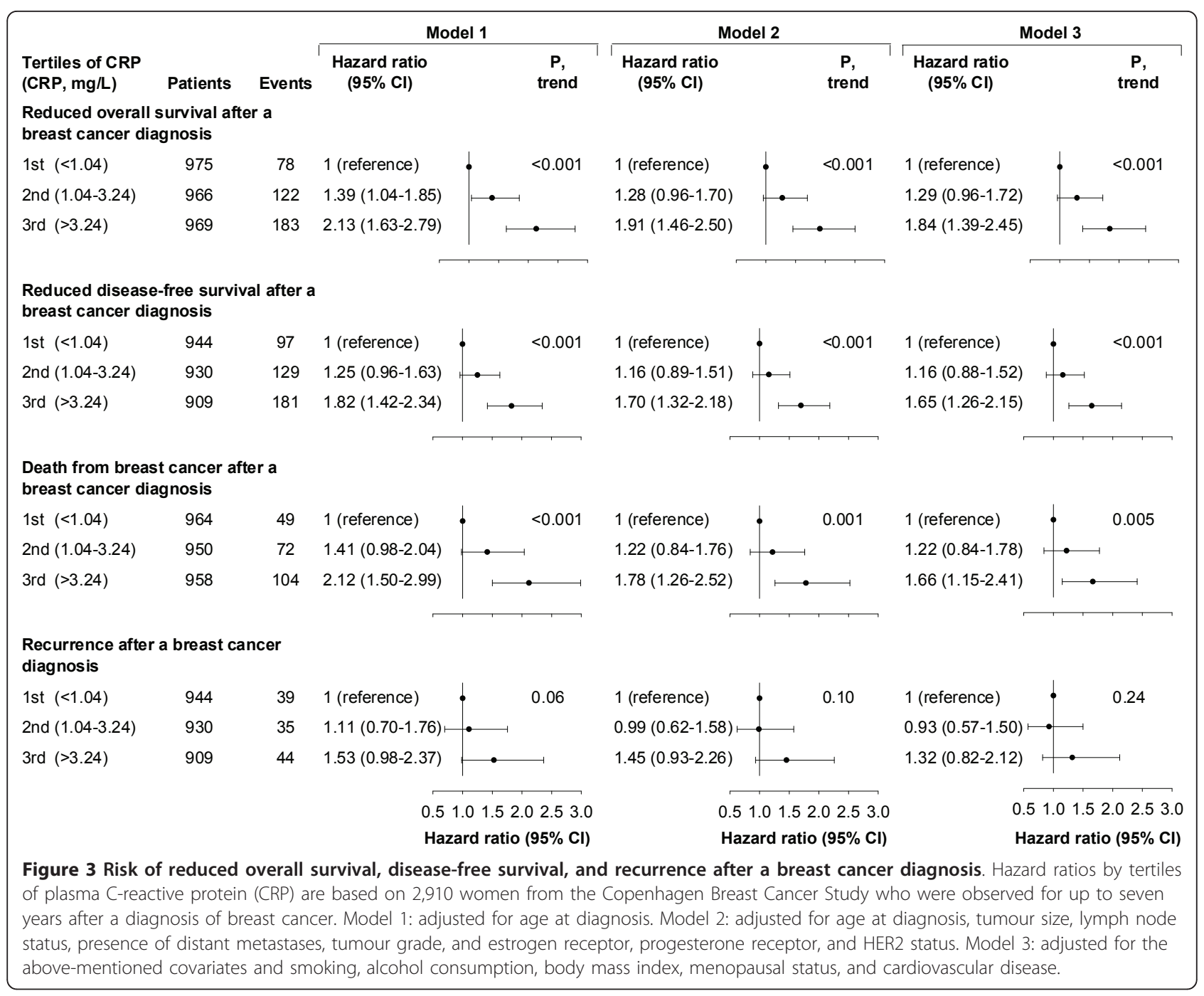

breast cancer and overall survival may not be breast cancer specific but can simply be due to CRP acting as a general marker of health and longevity. However, as $64 \%$ of the deaths in the present study were due to breast cancer, breast cancer was the leading cause of death in the present cohort. The fact that the observed association between elevated CRP levels and increased risk of death from breast cancer was of the same magnitude as the observed association between elevated CRP levels and overall survival suggest that CRP may indeed be associated with mortality through a breast-cancerrelated mechanism. Elevated plasma CRP is a wellknown marker of cardiovascular disease [31], which could imply that elevated CRP levels are associated with overall survival through the association with cardiovascular disease. However, the fact that only $11 \%$ of the deaths in the present cohort were due to cardiovascular diseases and the fact that we adjusted for cardiovascular disease at baseline limit the possibility that our finding on overall survival is severely confounded by deaths from cardiovascular disease.

Our findings of a positive association between elevated CRP levels and poor breast cancer prognosis are in contrast to negative studies with 110 to 300 patients $[25,26]$, but are indirectly supported by findings from other studies with 85 to 734 patients which did report a positive association [21-24]. The largest study so far which comprised approximately 700 women treated successfully for early stage breast cancer found that elevated levels of CRP measured two and a half years after the time of diagnosis were associated with reduced disease-free and overall survival [23]. Thus, the combined evidence from this and our own study suggests that elevated plasma CRP levels are associated with short-term as well as long-term prognosis after breast cancer. Whereas studies that measure CRP after diagnosis and treatment reflect long-term prognosis, studies that measure CRP at the time of diagnosis more likely reflect 


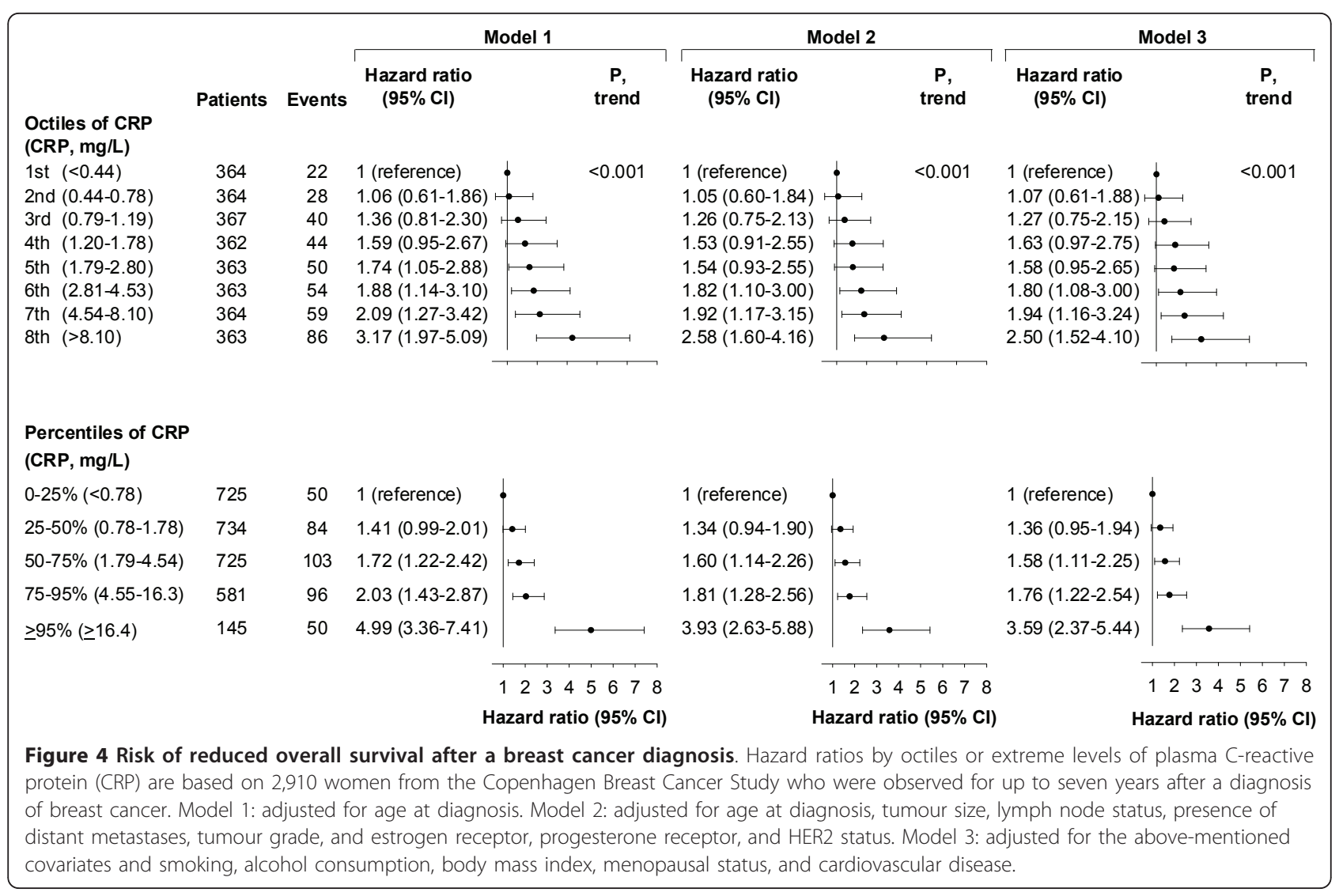

breast cancer specific survival. In fact, approximately $55 \%$ of the deaths, $60 \%$ of the deaths from breast cancer, and $68 \%$ of the recurrences in the present study occurred during the first two and a half years after a diagnosis of breast cancer. Therefore, our results may not be comparable to studies that have measured CRP after diagnosis and treatment.

The large sample size of our study allowed us to stratify for well-established prognostic tumour characteristics, and interestingly our data suggest that elevated CRP levels are associated with reduced overall survival irrespective of age at diagnosis, tumour size, lymph node status, presence of distant metastases, tumour grade, and estrogen receptor, progesterone receptor, and HER2 status. However, we observed a very strong association between elevated CRP levels and reduced overall survival among women with positive HER2 status which may warrant further investigation by future studies.

Dividing plasma CRP levels into octiles resulted in a stepwise increased risk of reduced overall survival, demonstrating the robustness of the observed association between elevated CRP levels and risk of reduced overall survival. Furthermore, we observed that compared to women with CRP levels in the 0 to $25 \%$ percentile $(\mathrm{CRP}<0.78 \mathrm{mg} / \mathrm{L})$, women with CRP levels $\geq 95 \%$ percentile ( $\geq 16.4 \mathrm{mg} / \mathrm{L}$ ) had a 3.5 -fold increased risk of reduced overall survival, suggesting that women with high CRP levels at the time of diagnosis have a particularly poor survival.

Although the age-adjusted HR for recurrence for the highest versus the lowest tertile of CRP was borderline significant, we did not detect a statistically significant association between elevated CRP levels and recurrence of breast cancer, which may suggest that no such association exists. However, as we only detected 118 recurrences in our study in contrast to 383 deaths, it is also possible that our statistical power was too small to detect an association. Therefore, other, larger studies are needed to answer unequivocally whether increased CRP levels at the time of diagnosis associate with recurrence of breast cancer or not.

As CRP is one of several acute-phase proteins, whose concentrations increase during acute or chronic inflammation, other inflammation-related biomarkers may also be associated with breast cancer prognosis. Prior studies have reported that lower serum albumin concentrations [25], elevated serum amyloid A concentrations [23], and elevated YKL-40 concentrations [32] are all associated with poor breast cancer prognosis.

So far, the present study is by far the largest study that has examined whether an association exists between 
Table 3 Risk of reduced overall survival by plasma levels of C-reactive protein and prognostic factors

\begin{tabular}{|c|c|c|c|c|c|c|c|}
\hline \multirow{3}{*}{ Stratification } & \multirow{3}{*}{ No. of patients } & \multirow{3}{*}{ No. of deaths } & \multicolumn{5}{|c|}{ Tertiles of C-reactive protein (mg/L) } \\
\hline & & & \multirow[t]{2}{*}{$P$, inter-action } & 1st $(<1.04)$ & 2nd (1.04 to 3.24$)$ & $3 \mathrm{rd}(>3.24)$ & $P$, trend \\
\hline & & & & \multicolumn{3}{|c|}{ Multifactor-adjusted hazard ratio $(95 \% \mathrm{Cl})$} & \\
\hline None & 2,910 & 383 & & 1.0 & 1.29 (0.96 to 1.72$)$ & $1.84(1.39$ to 2.45$)$ & $<0.001$ \\
\hline \multicolumn{8}{|l|}{ Age at diagnosis } \\
\hline$<57$ years & 964 & 74 & 0.40 & 1.0 & 0.97 (0.49 to 1.90$)$ & 1.69 (0.91 to 3.14) & 0.08 \\
\hline 57 to 69 years & 964 & 103 & & 1.0 & 1.76 (0.99 to 3.11$)$ & 2.53 (1.42 to 4.50$)$ & 0.002 \\
\hline$\geq 69$ years & 982 & 206 & & 1.0 & 1.18 (0.78 to 1.79$)$ & 1.59 (1.07 to 2.37$)$ & 0.01 \\
\hline \multicolumn{8}{|l|}{ Tumour size } \\
\hline$\leq 20 \mathrm{~mm}$ & 1,501 & 119 & 0.33 & 1.0 & 1.02 (0.62 to 1.68$)$ & 1.66 (1.03 to 2.68$)$ & 0.03 \\
\hline 20 to $50 \mathrm{~mm}$ & 1,409 & 264 & & 1.0 & 1.48 (1.02 to 2.15$)$ & $2.04(1.41$ to 2.95$)$ & $<0.001$ \\
\hline$>50 \mathrm{~mm}$ & 103 & 23 & & 1.0 & 2.74 (0.36 to 20.7) & $0.63(0.06$ to 6.61$)$ & 0.44 \\
\hline \multicolumn{8}{|l|}{ Lymph node status } \\
\hline Lymph node negative & 1,334 & 132 & 0.59 & 1.0 & 1.07 (0.65 to 1.76$)$ & $1.48(0.92$ to 2.38$)$ & 0.08 \\
\hline Lymph node positive & 1,335 & 191 & & 1.0 & 1.55 (1.01 to 2.38$)$ & 2.04 (1.33 to 3.12$)$ & 0.001 \\
\hline \multicolumn{8}{|l|}{ Distant metastases } \\
\hline No & 2,780 & 343 & 0.66 & 1.0 & 1.31 (0.96 to 1.78$)$ & 1.80 (1.33 to 2.44$)$ & $<0.001$ \\
\hline Yes & 40 & 23 & & 1.0 & 0.50 (0.01 to 21.0) & 15.6 (1.10 to 221) & 0.03 \\
\hline \multicolumn{8}{|l|}{ Tumour grade } \\
\hline Well differentiated & 685 & 50 & 0.69 & 1.0 & 0.74 (0.31 to 1.75$)$ & 1.49 (0.68 to 3.28$)$ & 0.21 \\
\hline Moderately differentiated & 1,262 & 154 & & 1.0 & 1.17 (0.74 to 1.88$)$ & 1.78 (1.14 to 2.77$)$ & 0.007 \\
\hline Poorly/un-differentiated & 505 & 92 & & 1.0 & 2.19 (1.16 to 4.14$)$ & $2.23(1.14$ to 4.39$)$ & 0.03 \\
\hline \multicolumn{8}{|l|}{ Estrogen receptor status } \\
\hline Positive & 2,233 & 234 & 0.83 & 1.0 & 1.07 (0.74 to 1.55$)$ & 1.59 (1.11 to 2.28$)$ & 0.005 \\
\hline Negative & 436 & 88 & & 1.0 & 2.07 (1.08 to 3.98) & 2.74 (1.44 to 5.19$)$ & 0.002 \\
\hline \multicolumn{8}{|l|}{ Progesterone receptor status } \\
\hline Positive & 1,383 & 87 & 0.60 & 1.0 & 1.83 (0.93 to 3.59$)$ & 2.37 (1.23 to 4.58$)$ & 0.01 \\
\hline Negative & 787 & 130 & & 1.0 & 1.47 (0.88 to 2.47$)$ & $2.22(1.33$ to 3.71$)$ & 0.002 \\
\hline \multicolumn{8}{|l|}{ HER2 status } \\
\hline Positive & 807 & 29 & 0.11 & 1.0 & 1.64 (0.34 to 7.96$)$ & 8.63 (2.04 to 36.4 ) & $<0.001$ \\
\hline Negative & 220 & 19 & & 1.0 & $1.24(0.24$ to 6.49$)$ & 1.29 (0.31 to 5.45$)$ & 0.74 \\
\hline
\end{tabular}

Blood samples were drawn and CRP measured at time of diagnosis of breast cancer.

Hazard ratios are multifactor-adjusted for age at diagnosis, tumour size, lymph node status, presence of distant metastases, tumour grade, and estrogen receptor, progesterone receptor, and HER2 status, smoking, alcohol consumption, body mass index, menopause status, and cardiovascular disease (Model 3), excluding the factor stratified for; however, age stratified analyses were still adjusted for age.

Due to differences in numbers of missing values for the covariates in question, numbers of patients and deaths do not necessarily add up to 2,910 and 383 , respectively.

CRP levels at the time of diagnosis and breast cancer prognosis. However, certain limitations must be acknowledged. Although we did adjust for potential confounders, we cannot exclude residual confounding by factors not taken into account, such as socioeconomic status, physical activity, and treatment, or by imperfectly or incompletely measured confounders. Since patients are referred to different treatment regimes on the basis of tumour characteristics, which we did adjust for, it seems unlikely that our results are confounded by differences in treatment. Also, we did not measure serum amyloid A, which has previously been associated with an even stronger increase in risk of death than CRP [23]. Finally, since the present study is a single-center study of Danish women diagnosed with breast cancer, our findings may not necessarily apply to other ethnic groups. Since our department for breast surgery treats all patients with breast cancer from a geographically well-defined area of Copenhagen regardless of severity or sub-diagnosis, our results may indeed be generalized to comparable populations of unselected patients with breast cancer.

\section{Conclusions}

In conclusion, in 2,910 Danish women with invasive breast cancer, elevated CRP levels at the time of diagnosis of breast cancer were associated with reduced overall and disease-free survival and with increased risk of 
death from breast cancer, independently of well-established prognostic tumour characteristics and lifestyle factors.

\section{Abbreviations}

Cl: confidence interval; CRP: C-reactive protein; HR: hazard ratio; ICD: International Classification of Diseases; WHO: World Health Organization

\section{Acknowledgements}

We are indebted to the staff and participants of the Copenhagen Breast Cancer Study for their important contributions. This work was supported by the Danish Medical Research Council; the Research Council at Herlev Hospital, Copenhagen University Hospital; and Chief Physician Johan Boserup and Lise Boserups Fund. The study sponsors had no role in the design and conduct of the study; in the collection, management, analysis, and interpretation of the data; or in the preparation, review, or approval of the manuscript.

\section{Author details}

'Department of Clinical Biochemistry Herlev Hospital, Copenhagen University Hospital, Herlev Ringvej 75, Herlev, DK-2730, Denmark. ${ }^{2}$ Faculty of Health Sciences, University of Copenhagen, Blegdamsvej 3, Copenhagen, DK-2200, Denmark. ${ }^{3}$ Department of Breast Surgery Herlev Hospital, Copenhagen University Hospital, Herlev Ringvej 75, Herlev, DK-2730, Denmark.

\section{Authors' contributions}

All authors contributed to the conception and design of the study. HF and SEB contributed to the acquisition of data. KHA performed the statistical analysis and drafted the manuscript. BGN, HF and SEB critically revised the manuscript. All authors read and approved the final manuscript.

\section{Competing interests}

The authors declare that they have no competing interests.

Received: 6 July 2010 Revised: 28 March 2011 Accepted: 3 June 2011 Published: 3 June 2011

\section{References}

1. Johnson AM: Amino acids, peptides, and proteins. In Tietz Textbook of Clinical Chemistry and Molecular Diagnostics.. 4 edition. Edited by: Burtis CA, Ashwood ER, Bruns DE. St. Louis, MO: Elsevier Saunders; 2006:555-556.

2. Pepys MB, Hirschfield GM: C-reactive protein: a critical update. J Clin Invest 2003, 111:1805-1812.

3. Heikkila K, Ebrahim S, Lawlor DA: A systematic review of the association between circulating concentrations of $C$ reactive protein and cancer. J Epidemiol Community Health 2007, 61:824-833.

4. Gauldie J, Richards C, Harnish D, Lansdorp P, Baumann H: Interferon beta 2/B-cell stimulatory factor type 2 shares identity with monocyte-derived hepatocyte-stimulating factor and regulates the major acute phase protein response in liver cells. Proc Natl Acad Sci USA 1987, 84:7251-7255.

5. Balkwill F, Mantovani A: Inflammation and cancer: back to Virchow? Lancet 2001, 357:539-545.

6. Colotta F, Allavena P, Sica A, Garlanda C, Mantovani A: Cancer-related inflammation, the seventh hallmark of cancer: links to genetic instability. Carcinogenesis 2009, 30:1073-1081.

7. Coussens LM, Werb Z: Inflammation and cancer. Nature 2002, 420:860-867.

8. Grivennikov $\mathrm{SI}$, Greten FR, Karin M: Immunity, inflammation, and cancer. Cell 2010, 140:883-899.

9. Mantovani A, Allavena P, Sica A, Balkwill F: Cancer-related inflammation. Nature 2008, 454:436-444

10. Allin KH, Bojesen SE, Nordestgaard BG: Baseline C-reactive protein is associated with incident cancer and survival in patients with cancer. J Clin Oncol 2009, 27:2217-2224.

11. Schmid M, Schneitter A, Hinterberger S, Seeber J, Reinthaller A, Hefler L: Association of elevated C-reactive protein levels with an impaired prognosis in patients with surgically treated endometrial cancer. Obstet Gynecol 2007, 110:1231-1236.
12. Polterauer S, Grimm C, Tempfer C, Sliutz G, Speiser P, Reinthaller A, Hefler LA: C-reactive protein is a prognostic parameter in patients with cervical cancer. Gynecol Oncol 2007, 107:114-117.

13. O'Dowd C, McRae LA, McMillan DC, Kirk A, Milroy R: Elevated preoperative C-reactive protein predicts poor cancer specific survival in patients undergoing resection for non-small cell lung cancer. J Thorac Oncol 2010, 5:988-992.

14. Roxburgh CS, McMillan DC: Role of systemic inflammatory response in predicting survival in patients with primary operable cancer. Future Oncol 2010, 6:149-163.

15. Das RL, Pathangey LB, Tinder TL, Schettini JL, Gruber HE, Mukherjee P. Breast-cancer-associated metastasis is significantly increased in a model of autoimmune arthritis. Breast Cancer Res 2009, 11:R56.

16. DeNardo DG, Coussens LM: Inflammation and breast cancer. Balancing immune response: crosstalk between adaptive and innate immune cells during breast cancer progression. Breast Cancer Res 2007, 9:212.

17. Ginestier C, Liu S, Diebel ME, Korkaya H, Luo M, Brown M, Wicinski J, Cabaud O, Charafe-Jauffret E, Birnbaum D, Guan JL, Dontu G, Wicha MS: CXCR1 blockade selectively targets human breast cancer stem cells in vitro and in xenografts. J Clin Invest 2010, 120:485-497.

18. Leek RD, Lewis CE, Whitehouse R, Greenall M, Clarke J, Harris AL: Association of macrophage infiltration with angiogenesis and prognosis in invasive breast carcinoma. Cancer Res 1996, 56:4625-4629.

19. Liao D, Luo Y, Markowitz D, Xiang R, Reisfeld RA: Cancer associated fibroblasts promote tumour growth and metastasis by modulating the tumour immune microenvironment in a 4T1 murine breast cancer model. PLoS One 2009, 4:e7965.

20. Mantovani A, Marchesi F, Porta C, Sica A, Allavena P: Inflammation and cancer: breast cancer as a prototype. Breast 2007, 16(Suppl 2):S27-S33.

21. Al Murri AM, Bartlett JM, Canney PA, Doughty JC, Wilson C, MCMillan DC: Evaluation of an inflammation-based prognostic score (GPS) in patients with metastatic breast cancer. Br J Cancer 2006, 94:227-230.

22. Albuquerque KV, Price MR, Badley RA, Jonrup I, Pearson D, Blamey RW, Robertson JF: Pre-treatment serum levels of tumour markers in metastatic breast cancer: a prospective assessment of their role in predicting response to therapy and survival. Eur I Surg Oncol 1995, 21:504-509.

23. Pierce BL, Ballard-Barbash R, Bernstein L, Baumgartner RN, Neuhouser ML, Wener MH, Baumgartner KB, Gilliland FD, Sorensen BE, McTiernan A, Ulrich CM: Elevated biomarkers of inflammation are associated with reduced survival among breast cancer patients. J Clin Oncol 2009, 27:3437-3444.

24. Williams MR, Turkes A, Pearson D, Griffiths K, Blamey RW: An objective biochemical assessment of therapeutic response in metastatic breast cancer: a study with external review of clinical data. Br J Cancer 1990, 61:126-132.

25. Al Murri AM, Wilson C, Lannigan A, Doughty JC, Angerson WJ, McArdle CS, McMillan DC: Evaluation of the relationship between the systemic inflammatory response and cancer-specific survival in patients with primary operable breast cancer. Br J Cancer 2007, 96:891-895.

26. Pasanisi P, Venturelli E, Morelli D, Fontana L, Secreto G, Berrino F: Serum insulin-like growth factor-I and platelet-derived growth factor as biomarkers of breast cancer prognosis. Cancer Epidemiol Biomarkers Prev 2008, 17:1719-1722

27. Moller S, Jensen MB, Ejlertsen B, Bjerre KD, Larsen M, Hansen HB, Christiansen P, Mouridsen HT: The clinical database and the treatment guidelines of the Danish Breast Cancer Cooperative Group (DBCG); its 30-years experience and future promise. Acto Oncol 2008, 47:506-524.

28. Kamstrup PR, Benn M, Tybjaerg-Hansen A, Nordestgaard BG: Extreme lipoprotein(a) levels and risk of myocardial infarction in the general population: the Copenhagen City Heart Study. Circulation 2008, 117:176-184.

29. Zacho J, Tybjaerg-Hansen A, Nordestgaard BG: C-reactive protein and allcause mortality-the Copenhagen City Heart Study. Eur Heart J 2010, 31:1624-1632.

30. Koenig W, Khuseyinova N, Baumert J, Meisinger C: Prospective study of high-sensitivity C-reactive protein as a determinant of mortality: results from the MONICA/KORA Augsburg Cohort Study, 1984-1998. Clin Chem 2008, 54:335-342. 
31. Zacho J, Tybjaerg-Hansen A, Jensen JS, Grande P, Sillesen H,

Nordestgaard BG: Genetically elevated C-reactive protein and ischemic vascular disease. N Engl J Med 2008, 359:1897-1908.

32. Johansen JS, Christensen IJ, Riisbro R, Greenall M, Han C, Price PA, Smith K Brunner N, Harris AL: High serum YKL-40 levels in patients with primary breast cancer is related to short recurrence free survival. Breast Cancer Res Treat 2003, 80:15-21.

doi:10.1186/bcr2891

Cite this article as: Allin et al: Elevated pre-treatment levels of plasma

C-reactive protein are associated with poor prognosis after breast cancer: a cohort study. Breast Cancer Research 2011 13:R55.

Submit your next manuscript to BioMed Central and take full advantage of:

- Convenient online submission

- Thorough peer review

- No space constraints or color figure charges

- Immediate publication on acceptance

- Inclusion in PubMed, CAS, Scopus and Google Scholar

- Research which is freely available for redistribution

Submit your manuscript at www.biomedcentral.com/submit 